\title{
Zest Anchors wird zu Zest Dental Solutions
}

Zest Anchors, eines der global führenden Unternehmen im Bereich Entwicklung und Herstellung von Prothesenbefestigungen,

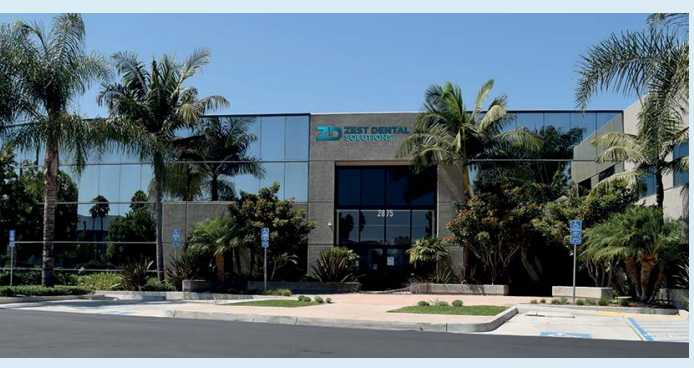

verkündet die Änderung des Firmennamens in Zest Dental Solutions, um so die bisherige Entwicklung des Unternehmens sowie dessen Zukunftsvision widerzuspiegeln. Seit mehr als 40 Jahren verbessert das Unternehmen die Lebensqualität zahnloser Patienten weltweit mit innovativen und funktionalen Prothetiklösungen. Das Vorzeigeprodukt, das LOCATOR-Attachment-System, gilt für die Implantatindustrie sowie Behandler und Patienten bis zum heutigen Tag als zuverlässigste Marke für Deckprothesen. Das Unternehmen machte sich zum Ziel, kontinuierlich weitere Pa- tientenlösungen anzubieten und präsentierte neben einer Implantat-Reihe mit niedrigem Durchmesser das CHAIRSIDEProduktportfolio - bestehend aus zahnärztlichen Instrumenten und Materialien für die Herstellung und Anpassung von Deckprothesen - sowie das neue LOCATOR R-Tx Removable Attachment-System. Das Unternehmen wird mit dem LOCATOR F-Tx Fixed Attachment System, dessen Launch für das 4. Quartal 2016 vorgesehen ist, auch in den Bereich der festsitzenden Versorgungen des vollständigen Zahnbogens einsteigen. Anfang 2016 wurde Danville Materials erworben, ein führender Hersteller von restaurativen Verbrauchsmaterialien, Kleingeräten und minimalinvasiven Behandlungslösungen wie Perioscopy. Dieser Kauf positioniert Zest als ein Unternehmen, das Produktlösungen für ein ganzes Kontinuum der Patientenversorgung anbietet - von zahnerhaltenden Lösungen bis zur Behandlung vollständiger Zahnlosigkeit.

\author{
Nach einer Pressemitteilung der \\ Zest Dental Solutions, USA - Carlsbad
} www.zestdent.com. 